\title{
Cationic lipid-based nanoparticles mediate functional delivery of acetate to tumor cells in vivo leading to significant anticancer effects
}

This article was published in the following Dove Press journal:

International Journal of Nanomedicine

8 September 2017

Number of times this article has been viewed

\author{
Leigh P Brody ${ }^{1, *}$ \\ Meliz Sahuri-Arisoylu',* \\ James R Parkinson' \\ Harry G Parkes ${ }^{2}$ \\ Po Wah So ${ }^{3}$ \\ Nabil Hajji ${ }^{4}$ \\ E Louise Thomas' \\ Gary S Frost ${ }^{5}$ \\ Andrew D Miller,* \\ Jimmy D Bell ${ }^{1, *}$
}

'Department of Life Sciences, Faculty of Science and Technology, University of Westminster, ${ }^{2} \mathrm{CR}$-UK Clinical MR Research Group, Institute of Cancer Research, Sutton, Surrey, ${ }^{3}$ Department of Neuroimaging, Institute of Psychiatry, Psychology and Neuroscience, King's College London, ${ }^{4}$ Department of Medicine, Division of Experimental Medicine,

Centre for Pharmacology \&

Therapeutics, Toxicology Unit, Imperial College London, ${ }^{5}$ Faculty of Medicine, Nutrition and Dietetic Research Group, Division of Diabetes, Endocrinology and Metabolism,

Department of Investigative Medicine, Imperial College London, Hammersmith Hospital, 'Institute of Pharmaceutical Science, King's College London, London, UK

*These authors contributed equally to this work

Correspondence: Jimmy D Bell Department of Life Sciences, Faculty of Science and Technology, University of Westminster, II 5 New Cavendish Street, London WIW 6XH, UK

Email j.bell@westminster.ac.uk
Abstract: Metabolic reengineering using nanoparticle delivery represents an innovative therapeutic approach to normalizing the deregulation of cellular metabolism underlying many diseases, including cancer. Here, we demonstrated a unique and novel application to the treatment of malignancy using a short-chain fatty acid (SCFA)-encapsulated lipid-based delivery system liposome-encapsulated acetate nanoparticles for cancer applications (LITA-CAN). We assessed chronic in vivo administration of our nanoparticle in three separate murine models of colorectal cancer. We demonstrated a substantial reduction in tumor growth in the xenograft model of colorectal cancer cell lines HT-29, HCT-116 p53+/+ and HCT-116 p53-/-. Nanoparticle-induced reductions in histone deacetylase gene expression indicated a potential mechanism for these anti-proliferative effects. Together, these results indicated that LITA-CAN could be used as an effective direct or adjunct therapy to treat malignant transformation in vivo.

Keywords: lipid-based nanoparticles, liposomes, cancer, short-chain fatty acids, epigenetics

\section{Plain language summary}

Non-digestible carbohydrates inhibit the growth of certain cancers. These effects are linked to short-chain fatty acids (SCFAs), such as acetate, which are produced by the breakdown of non-digestible carbohydrates in the gut. The exact mechanisms by which SCFAs inhibit cancer growth are unknown, and finding out is complicated by their large number of biological functions, including sugar, fat and cholesterol metabolism and secretion of appetite-controlling hormones. In addition, the use of SCFAs as a cancer therapy is limited by the difficulty in safely delivering sufficiently high levels to specific sites within the body.

In this study, we packaged the SCFA acetate within special nanoparticles, termed liposomeencapsulated acetate nanoparticles for cancer applications (LITA-CAN). We showed that LITA-CAN delivery leads to a substantial reduction in tumor growth in three different mouse models of colorectal cancer. In addition, we showed that LITA-CAN reduce expression of histone deacetylase (HDAC) proteins, key enzymes involved in cell division and growth. Together, these results indicated that LITA-CAN could be used as an effective therapy to treat malignant transformation.

\section{Introduction}

Dietary supplementation with fermentable carbohydrates (FC) has been shown to attenuate malignant growth in certain cancers. ${ }^{1,2}$ These effects are linked to the production of short chain fatty acids (SCFAs), predominantly acetate, propionate and butyrate, which are generated by fermentation of FC by microbiota in the colon. ${ }^{3}$ The antitumorigenic effects attributed to SCFAs are, in turn, thought to arise from their altering 
expression of histone deacetylase (HDAC) enzymes. ${ }^{1,4}$ HDACs are important epigenetic markers that play a key role in modulating the expression of genes involved in cellular proliferation, apoptosis and differentiation. ${ }^{5,6}$ As such, they represent an integrated target for cancer therapy, with the ineffectual metabolism of SCFA in cancer cells hypothesized to lead to an accumulation of acetyl-CoA in the nucleus, where it functions as an HDAC inhibitor. ${ }^{7}$

Previous studies have attempted to target and disrupt the altered cellular metabolism in colonic cancer cells via supplementation with SCFA., ${ }^{1,8}$ However, the ubiquitous role of SCFAs in metabolism, combined with a short tissue half-life, an adversely low $\mathrm{pH}$ in solution and the nontargeted nature of oral and peripheral administrations limits their therapeutic potential. ${ }^{9}$ Indeed, reductions in tumor growth, mediated by butyrate inhibition of HDAC enzymes, are limited by a short half-life and first-pass hepatic clearance following peripheral delivery. ${ }^{10}$ Liposomal encapsulation of therapeutic agents addresses a number of these issues by improving bioavailability, extending treatment effects and potentially reducing dosing. ${ }^{11}$ Liposomes are an effective tool for drug conveyance, in part because they can be engineered with properties that enhance functional delivery to specific locations. ${ }^{12}$ Polyethylene glycol (PEG)-lipids can be incorporated into the lipid bilayer to enhance biocompatibility and increase circulation times. ${ }^{13}$ This modification is especially effective in passively targeting malignant tissue, with increased liposomal uptake through leaky tumor microvasculature and prolonged retention time due to reduced lymphatic drainage occurring via the enhanced permeability and retention (EPR) effect. ${ }^{14,15}$

We have previously demonstrated that peripheral administration of PEGylated nanoparticles containing anticancer Survivin siRNA leads to a significant reduction in tumor growth in a murine xenograft model of breast cancer. ${ }^{16}$ In addition, we have formulated lipid-encapsulated acetate (LITA) nanoparticles which, following peripheral delivery in obese mice, resulted in improvements in a wide range of metabolic outcomes. ${ }^{17}$ Here, we encapsulated acetate within specifically formulated liposome-encapsulated acetate nanoparticles for cancer applications (LITA-CAN) and assessed their effects in three xenograft murine models of human colorectal cancer.

\section{Material and methods Materials and experimental animals}

All in vivo experiments were carried out in compliance with the Animals (Scientific Procedure) Act 1986. Mice were supplied by Harlan, UK. Mice were housed four per cage in a temperature-controlled room at $\sim 25^{\circ} \mathrm{C}$ with alternating 12-hour light/dark periods (light: 8:00-20:00) with ad libitum access to food and water. All experiments were carried out with ethical approval from the local ethical review committee of Imperial College London in compliance with the UK Home Office Animals (Scientific Procedure) Act 1986 under the project license $70 / 6656$.

\section{Preparation of liposome nanoparticles}

Nanoparticle design was based upon our previous studies, where PEGylated liposomes were formulated encapsulating acetate for uptake in the peripheral tissues ${ }^{17}$ or specifically designed for preferential uptake in xenograft tumors. ${ }^{16}$ Liposomes were prepared by the thin film hydration method, ${ }^{18}$ with constituent molar ratios depending on the proportion of $N^{1}$-cholesteryloxycarbonyl-3,7-diazanonane-1,9-diamine (CDAN)/1,2-distearoyl-sn-glycero-3-phosphocholine (DSPC)/1,2-distearoyl-sn-glycero-3-phosphoethanolamine$N$-methoxy PEG ${ }^{2000}$ (DSPE-PEG ${ }^{2000}$ )/cholesterol (Chol). Optimization of components was performed to maximize size and acetate encapsulation. For xenograft delivery, the molarity percentage of DSPE-PEG ${ }^{2000}$ in the liposome membrane was increased from $1 \%$ to $5 \%$ compared to previous constitutions (Figure S1), to prolong circulation and enable preferential accumulation in tumor tissue via the EPR effect. Liposomes were prepared with either acetate (1 M, pH 2.3) to form LITA-CAN or 4-(2-hydroxyethyl)1-piperazineethanesulfonic acid (HEPES; $4 \mathrm{mM}, \mathrm{NaCl}$ $135 \mathrm{mM}, \mathrm{pH}$ 6.5) for use as control. The lipid layer was sonicated with acetate or HEPES for 1 hour at $30^{\circ} \mathrm{C}$ in the dark, buffered to a $\mathrm{pH}$ of 7.0 and dialyzed overnight using the Float-A-Lyzer G2 device. Particle size was determined using a Malvern Zetasizer system (Malvern Instruments, Malvern, UK). LITA-CAN were constituted with $1 \%$ rhodamine (LITA-CAN-Rhd; Figure S1) for imaging purposes.

\section{Quantification of acetate inside LITA-CAN}

The presence and concentration of acetate within LITA-CAN were determined using ${ }^{1} \mathrm{H}$ NMR spectroscopy. ${ }^{17}$

\section{Tumor efficacy and tissue processing}

Xenograft models from three different colorectal cancer cell lines were investigated to explore the therapeutic potential of LITA-CAN; HT-29, HCT-116 wild-type p53 (HCT 116 p53+/+) and HCT-116 isogenic knocked-out p53 (HCT 116 p53-/-). The HT-29 cells were obtained from ATCC Cell Lines (LGC Standards, Middlesex, UK). HCT-116 p53+/+ and HCT-116 p53-/- lines were provided by Professor 
Joseph Bertarnd, Karolinska Institutet, Sweden, via Bert Vogelstein, Johns Hopkins Oncology Centre. Ethical and legal approval was obtained for all cell line usage from the local ethical review committee of Imperial College London in accordance with the Human Tissue Act of 2004. Cell lines were cultured in Dulbecco's Modified Eagle's Medium (DMEM; Sigma-Aldrich Co., St Louis, MO, USA), supplemented with 10\% fetal calf serum (FCS; SigmaAldrich Co.) in an incubator at $37^{\circ} \mathrm{C}$ with $5 \% \mathrm{CO}_{2}$. Cells were maintained every 3-5 days while never exceeding 15 passages. For generating luciferase-stable clones from HCT-116 p53+/+ and HCT-116 p53-/- for in vivo visualization, cell lines were transfected using polyethylenimine (PEI) polymers mixed with the pGL4.20 [luc2/Puro] Vector (Promega Corporation, Fitchburg, WI, USA) in a 4:1 ratio. Stable clones from each cell line (HCT-116 p53+/+ and HCT-116 p53-/-) were selected in puromycin for 2 weeks. To confirm the success of stable clones and check luciferase expression levels, Steady-Glo Luciferase Assay System (Promega Corporation) was used. Tumorigenicity was established in all models by subcutaneously injecting $2.5 \times 10^{5}$ cells of colon cancer cell line into the left flank of 6- to 8-week-old nude mice (BALB/c nu/nu). Confirmation of subcutaneous nude mouse xenograft tumors required 14-20 days.

\section{In vivo distribution of LITA-CAN-Rhd in HT-29 xenograft mice}

Once tumors were palpable, HT-29 animals were injected intraperitoneally (i.p.) with LITA-CAN-Rhd $(200 \mu \mathrm{L}$, $2.6 \mathrm{mg} / \mathrm{kg}$ of body weight, $\mathrm{n}=3$ ). After 2 hours of administration, tissue samples were collected and frozen at $-80^{\circ} \mathrm{C}$. A cryostat (Olympus Corporation, Tokyo, Japan) was used to generate $7 \mu \mathrm{m}$ slices and subsequently stained with $4^{\prime}, 6$ diamidino-2-phenylindole (DAPI). Images were obtained using an Olympus IX71 microscope (Leica Microsystems, Wetzlar, Germany).

\section{Effects of LITA-CAN administration on xenograft tumor metastasis}

Mice were injected i.p. with LITA-CAN or controls $(2.6 \mathrm{mg} / \mathrm{kg}$, $200 \mu \mathrm{L}$ volume) every 3 days for 4 weeks in each of the three xenograft models: HT-29 (n=6/group), HTC-116 p53+/+ ( $\mathrm{n}=8$ /group) and HTC-116 p53-/- $(\mathrm{n}=8$ /group). Tumor volume was monitored every $2-4$ days by a caliper to determine the volume estimated assuming an ellipsoid shape using the following equation: $V=L \times W \times D \times \pi / 6$. After 4 weeks, the tumors were excised, weighed and portioned to either formalin solution (Sigma-Aldrich Co.) for histological evaluation or snap frozen in liquid nitrogen and stored at $-80^{\circ} \mathrm{C}$ for subsequent extraction of mRNA.

\section{Hepatic HDAC expression}

Western blot was performed on liver samples from HT-29 mice to examine the effects of LITA-CAN on expression of class I, II and IV HDAC proteins. Raw signals for each were quantified and background subtracted using the Li-COR Odyssey software (LI-COR Biotechnology; Lincoln, NE, USA) and normalized to $\beta$-actin. Quantification of protein expression was performed using ImageJ software $(\mathrm{NIH}$, Maryland, USA).

\section{Tumor assessment by bioluminescent imaging luciferase}

Luminescent visualization of tumors in HCT-116 p53 wild type and knockout animals was carried out at week 3 using a IVIS 2000 small-animal in vivo imaging system and an IVIS 200 cooled CCD camera system (Xenogen Corp., Almeda, CA, USA) and IGOR software (Wavemetrics Corporation, Portland, OR, USA). Animals received a $100-\mu \mathrm{L}$ i.p. injection of D-luciferin (15 mg/mL; Gold Biotechnology, St Louis, MO, USA) under 1\%-2\% inhaled isoflurane anesthesia. An average of 10 kinetic bioluminescent acquisitions were collected between 0 and 30 minutes after substrate injection to confirm a peak photon emission recorded as maximum photon efflux per second. Data analyses and background correction were carried out using total photon flux emission (photons/s) in a region of interest (ROI) covering the entire xenograft tumor region.

\section{Statistical analysis}

Unless otherwise noted, all statistical analyses were performed using GraphPad Prism (La Jolla, CA, USA). Data are presented as mean \pm standard deviation (SD). Statistical significance was calculated with Student's $t$-test, two-way analysis of variance (ANOVA) with Bonferroni post-hoc test for multiple comparisons and generalized estimating equation (GEE) analysis where appropriate.

\section{Results}

\section{Nanoparticle preparation, quantification of size and encapsulated acetate concentration}

Liposome size and acetate encapsulated concentrations for nanoparticles of CDAN/DSPC/Chol/DSPE-PEG ${ }^{2000}$ variants are shown in Table S1. LITA and corresponding control nanoparticles were formulated using a CDAN/DSPC/Chol/ DSPE-PEG ${ }^{2000}$ molar ratio of 32:32:31:5. LITA-CAN-Rhd 
nanoparticles contained 1\% of 1,2-dioleoyl-sn-glycero-3phosphoethanolamine- $N$-(lissamine rhodamine B sulfonyl) (DOPE-rhodamine) component, with 1\% less cholesterol (32:32:30:5:1). Zetasizer analysis of nanoparticles revealed comparable sizes between LITA $\left(108.2 \pm 3.3 \mathrm{~nm}, 25^{\circ} \mathrm{C}\right)$ and corresponding control nanoparticles $\left(100.7 \pm 2.1 \mathrm{~nm}, 25^{\circ} \mathrm{C}\right)$. ${ }^{1} \mathrm{H}$ NMR analysis revealed that the optimal encapsulated acetate concentration for LITA liposomes was $6.13 \mathrm{mM}$.

\section{In vivo distribution of LIP-CAN-Rhd}

Tumorigenicity in nude mice (BALB/c nu/nu) was established by injecting colon cancer cell line HT-29. Once tumors were palpable, animals were injected i.p. with LITA-CANRhd $(200 \mu \mathrm{L}, 2.6 \mathrm{mg} / \mathrm{kg} ; \mathrm{n}=3)$. After 2 hours, tissue samples were collected, frozen and $7 \mu \mathrm{m}$ slices generated using a cryostat. A nuclear stain (DAPI) was initially allied to the microscope slide to ensure the region being observed was in fast tissue (Figure 1A), and a rhodamine-specific filter was used to confirm the presence of fluorescent-labeled LITACAN-Rhd in the tumor (Figure 1B).

\section{Effects of LITA-CAN delivery in an HT-29 colorectal cancer xenograft tumor model} Compared to control nanoparticles, LITA-CAN delivery led to a significant reduction in tumor size (LITA-CAN:
106.8 $\pm 113.3 \mathrm{~mm}^{2}$ vs control: $269.7 \pm 87.46 \mathrm{~mm}^{2}, P<0.05$; Figure $1 \mathrm{C}$ and D), weight (LITA-CAN: $0.046 \pm 0.032 \mathrm{mg}$ vs control: $0.33 \pm 0.04 \mathrm{mg}, P<0.01$; Figure $1 \mathrm{E})$ and volume after 4 weeks of therapy (LITA-CAN: $123.0 \pm 118.7 \mathrm{~mm}^{3}$ vs control: $254.2 \pm 93.6 \mathrm{~mm}^{3}, P<0.05$; Figure $1 \mathrm{~F}$ ).

\section{Hepatic HDAC expression}

Quantitative reverse transcription polymerase chain reaction (RT-PCR) of xenograft liver mRNA revealed a clear downregulation of Class I HDACs in LITA-CAN-injected animals, including HDAC-1 $(P<0.05)$, HDAC-2 $(P<0.05)$, HDAC-3 $(P<0.001)$ and HDAC-4 $(P<0.01)$ compared to controls (Figure 2). Sirtuins (SirTs) were also affected by LITA administration with a significant reduction in SirT1 $(P<0.05$; Figure 2).

\section{Effects of LITA-CAN delivery in an HCT II 6 p53+/+ and HCT I I 6 p53-/- colorectal cancer xenograft tumor model}

No differences in tumor size were recorded between LITACAN and controls in either HCT-116 p53+/+ $(P=0.27$; Figure 3A) or HCT-116 p53-/- $(P=0.41$; Figure 3D) mice, with similar nonsignificant trends toward reduced growth (HCT-116 p53+/+: $P=0.20$, Figure 3B; HCT-116 p53-/-: $P=0.61$, Figure $3 \mathrm{E})$. Regarding luciferase monitoring of
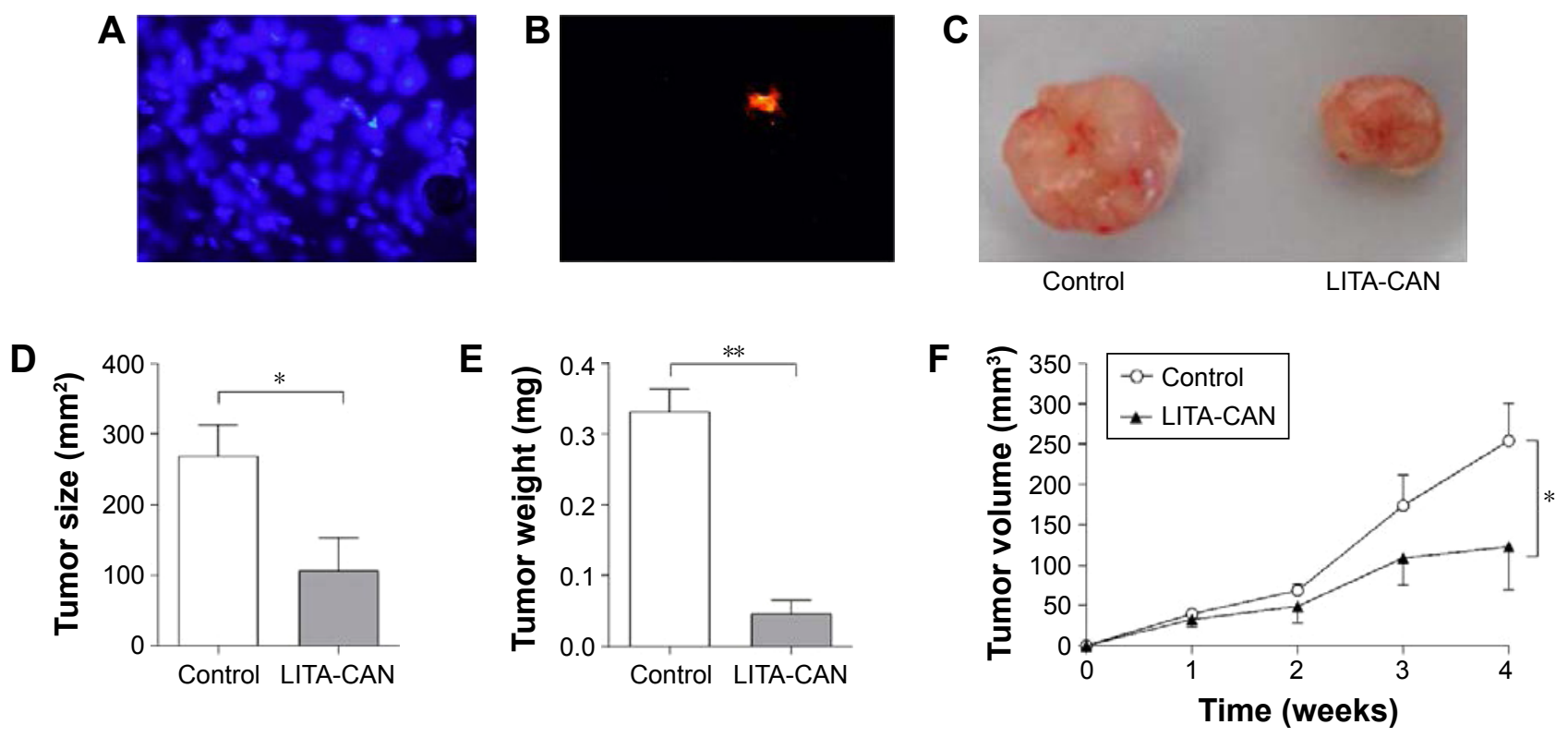

Figure I LITA-CAN-Rhd localization and the effects of LITA-CAN in an HT-29 xenograft model of colorectal cancer.

Notes: LITA-CAN-Rhd identification in the xenograft tumor by histological fluorescence imaging. (A) DAPI staining confirmed fast tissue. (B) Rhodamine-specific filter. The effects of LITA-CAN administration on HT-29 derived xenograft tumor metastasis; BALB/c nu/nu mice received an i.p. injection (2.6 mg/kg) of either LITA-CAN or control liposomes every 3 days for a 4 -week period. Tumors were extracted and weighed at the end of the 4 weeks study. (C) Representative tumors extracted from LITACAN and control mice. (D) Tumor size at 4 weeks. (E) Tumor weight. (F) Tumor volume as measured by a caliper over the 4 weeks. Data are presented as mean \pm SEM and analyzed using Student's $t$-test or GEE (GraphPad Prism). $n=6 /$ group. $* P<0.05$ and $* * P<0.01$. (A and $\mathbf{B}$ ) Magnification $\times 100$.

Abbreviations: LITA-CAN-Rhd, LITA-CAN constituted with I\% rhodamine; LITA-CAN, liposome-encapsulated acetate nanoparticles for cancer applications; DAPI, 4',6diamidino-2-phenylindole; i.p., intraperitoneally; SEM, standard error of the mean; GEE, generalized estimating equation. 


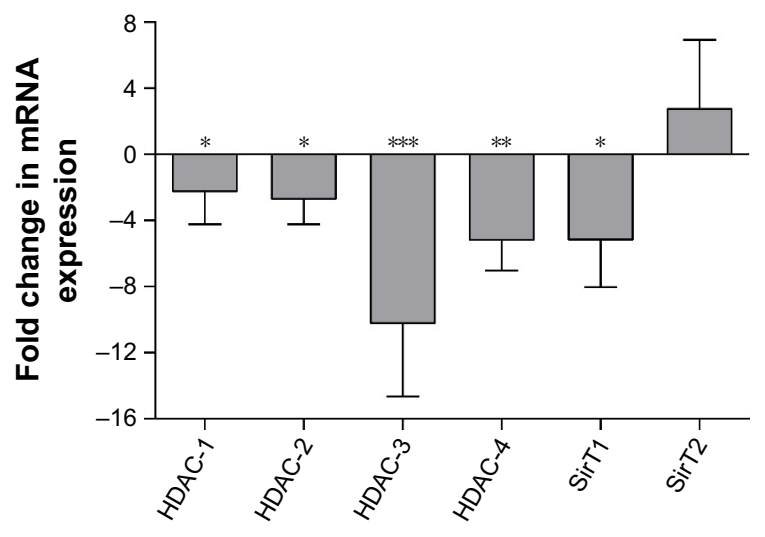

Figure 2 The effects of LITA-CAN on liver mRNA expression in an HT-29 xenograft model of colorectal cancer.

Notes: Quantitative changes in the expression of xenograft tumor liver mRNA at the end of 4 weeks of chronic LITA-CAN administration (i.p. injection every 3 days for 4 weeks of LITA-CAN or control $(2.6 \mathrm{mg} / \mathrm{kg})$ in an HT-29 xenograft model of colorectal cancer). Data represent fold change observed in LITA-CAN animals compared to controls; mRNA expression normalized $\beta$-actin. Quantification of protein expression was performed using ImageJ software. Data are presented as mean \pm SEM and analyzed by Student's $t$-test (GraphPad Prism). $n=6 /$ group. $* P<0.05$, $* * P<0.01$ and $* * * P<0.001$.

Abbreviations: LITA-CAN, liposome-encapsulated acetate nanoparticles for cancer applications; i.p., intraperitoneally; SEM, standard error of the mean. tumor size, LITA-treated groups again showed nonsignificant trends toward reduced luminescence compared to the control groups (HCT-116 p53+/+: $P=0.24$, Figure 3C; HCT-116 p53-/-: $P=0.74$, Figure 3F). Representative bioluminescence images are shown in Figure S2.

\section{Discussion}

Here, we described the preparation and therapeutic application of acetate encapsulating LITA-CAN liposomes to correct the impaired cellular metabolism associated with malignancy. Our data show that systemic administration of LITA-CAN leads to their accumulation in tumor tissue and, in the HT-29 murine xenograft model of colorectal cancer, mediates a reduction in growth. Furthermore, LITA-CANinduced alterations in HDAC and SirT mRNA expression indicate a potential epigenetic mechanism responsible for these beneficial effects.

Our initial work developing liposome formulations revealed that the percentage molarity of each component
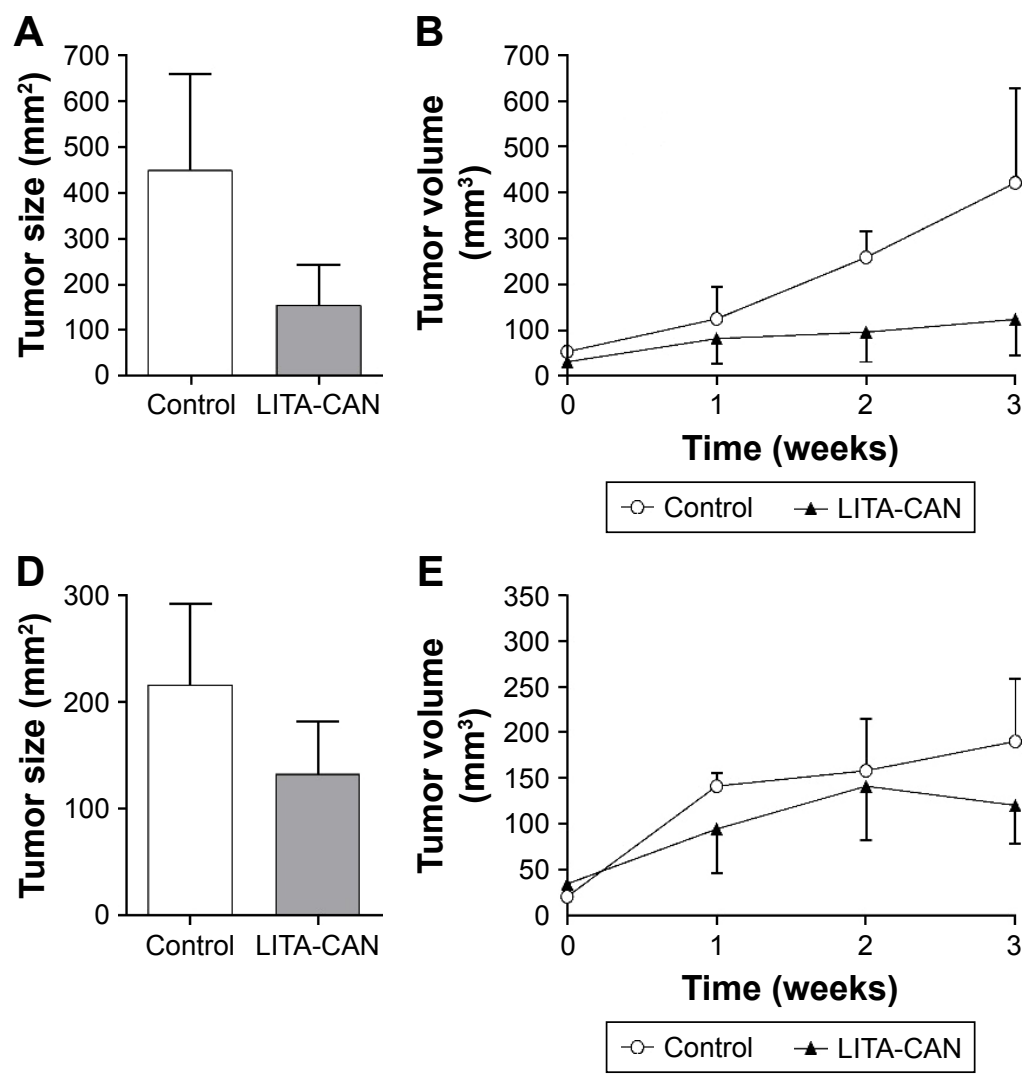
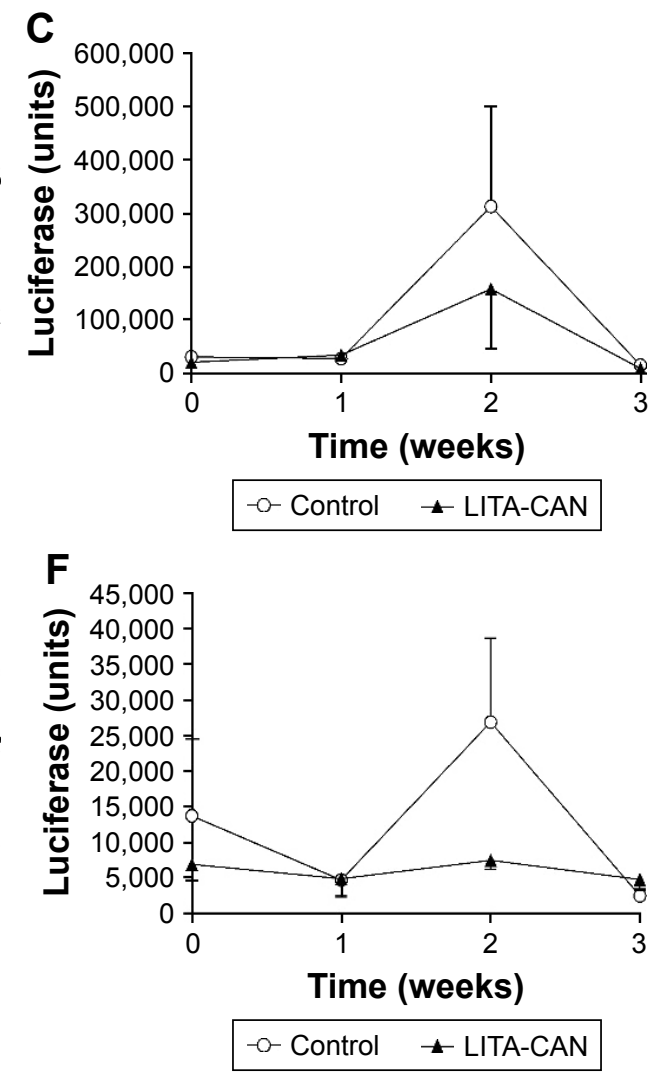

Figure 3 The effects of LITA-CAN administration on size, volume and luminescence in HTC-II6 p53+/+ and HTC-II6 p53-I- xenograft models of colorectal cancer. Notes: The effects of LITA-CAN administration on HTC-II6 p53+/+ (A-C) and HTC-II6 p53-/- (D-F) derived xenograft tumor metastasis; BALB/c nu/nu mice received an i.p. injection $(2.6 \mathrm{mg} / \mathrm{kg})$ of either LITA-CAN or control liposomes every 3 days for a 3 -week period. Tumors were extracted and weighed at the end of the 3 weeks study; tumor size at 3 weeks in (A) HTC-II6 p53+/+ and (D) HTC-116 p53-/-. Tumor growth in (B) HTC-II6 p53+/+ and (E) HTC-II6 p53-/-. Luciferase activity in (C) HTC-II 6 p53+/+ and (F) HTC-II6 p53-l-. Data are presented as mean \pm SEM and analyzed by two-way ANOVA with Bonferroni post hoc test or student's unpaired $t$-test. $\mathrm{n}=8 /$ group.

Abbreviations: LITA-CAN, liposome-encapsulated acetate nanoparticles for cancer applications; i.p., intraperitoneally; SEM, standard error of the mean; ANOVA, analysis of variance. 
plays an integral role in optimizing both the nanoparticle size and the permissible concentration of encapsulated acetate. In addition to maximizing the concentration of encapsulating acetate, LITA-CAN were specifically formulated with an increased 5\% DSPE-PEG ${ }^{2000}$ to promote greater systemic stability, longer circulation times and increased tumor uptake. ${ }^{19}$ Furthermore, heavily PEGylated liposomes $100 \mathrm{~nm}$ in diameter, such as LITA-CAN, are particularly well suited to enter tumor volumes via the EPR effect. ${ }^{14}$ This increased molarity percentage of DSPE-PEG ${ }^{2000}$ and apposite size led to a preferential accumulation of LITA-CAN in the tumor tissue. In addition to these data, our previous work has confirmed the integrity of the LITA nanoparticle complex to successfully and securely encapsulate acetate. In the same study, toxicological assessment of liver and adipocyte structure and function revealed no morphological abnormalities, inflammation or changes in the size or volume of adipocytes, following in vivo LITA delivery. ${ }^{17}$

Acetate plays a central role in cellular metabolism, functioning as a key intermediate in the synthesis of fatty acids and cholesterol in the liver. Furthermore, oxidation of acetate derived from carbohydrates, fats and proteins is the initial step in the tricarboxylic acid (TCA) cycle, a fundamental energy producing pathway in all aerobic organisms. ${ }^{20}$ While oxidative phosphorylation generates the majority of energy required in normal differentiated cells, cancer cells rely primarily on aerobic glycolysis, a metabolic shift termed the Warburgh effect that comprises attenuation of energy production via mitochondrial beta-oxidation. ${ }^{21}$ This defective energy metabolism, linked to impaired mitochondrial function, characterizes nearly all tumors, and offers the potential for effective targeted therapy. ${ }^{22-24}$

Within malignant cells, the inhibition of glucose uptake and metabolism by products of SCFA breakdown are thought to preferentially inhibit aerobic glycolysis and suppress uncontrolled cell growth. ${ }^{22,25}$ The anti-proliferative effects of SCFA have been linked to the enteric generation of acetate in several models of malignancy, ${ }^{1,4}$ with additional studies further linking these changes to SCFA-induced alterations in the activities of HDAC enzymes. ${ }^{5,10}$ In line with these data, we demonstrated that LITA-CAN administration leads to a significant reduction in the tumor growth and a concomitant reduction in the selected HDAC expression. The decreased expression of HDAC SirT1 is of particular note given its established role as an inhibitor of apoptosis and promoter for tumorigenesis. ${ }^{26}$ Furthermore, the observed reduction in SirT1 coupled with no effect on SirT2 expression mirrors in vitro data examining the effects of acetate and dichloroacetate (DCA), an analog of acetate with significant antitumor effects..$^{27} \mathrm{DCA}$ is proposed to inhibit pyruvate dehydrogenase kinase, shifting metabolism from glycolysis to oxidative phosphorylation, thus diminishing the Warburg effect. ${ }^{27}$ This in turn creates a substantial increase in the reactive oxygen species (ROS) production and subsequent cell death, ${ }^{28}$ and it is conceivable that LITA-CAN work in a comparable manner.

Taken together, these data indicate the anti-proliferative effects of our nanoparticle therapy are underpinned by the suppression of HDAC enzymes. However, given the ubiquitous role of acetate in cellular metabolism, the influence of acetate-induced metabolic reprogramming should not be overlooked. ${ }^{24,29}$ The LITA-CAN-induced attenuation of tumor growth in the HT-29 animals was not instantaneous with differential growth rates only apparent 3 weeks post commencement of administration. This suggests that acetate needs to be present for a significant period of time, saturating the tumor environment, before cellular responses can take place. Finally, we noted that HT-29 and HCT-116 p53+/+ xenografts appear more substantially affected by LITA-CAN administration than HCT-116 p53-/-, suggesting a role for $\mathrm{p} 53$ in LITA-CAN-induced anticancer effects. Individual colorectal cancer cell lines are characterized by their surface markers and colony morphology, which can self-renew and differentiate into multiple lineages. HCT-116 is a highly aggressive cell line that shows no ability to differentiate and does not express CDX-1, an intestine-specific transcription factor, while HT-29 has an intermediate capacity to differentiate. ${ }^{30}$ Indeed, the aggressive nature of the HCT-116 cell line resulted in necrotic tumors at the end of the third week of experimentation. As a result, this required animals to be culled and prevented our assessment of the effects of LITA-CAN into a fourth week in this model. Additional work into the exact mechanism by which LITACAN exerts its anti-proliferative effects will be required to understand the differing response we recorded in individual xenograft models.

Given the heterogeneity in cancer pathophysiology, it is important to note that the anti-tumorigenic effects of acetate we and others have observed in colonic cell lines may not be universally applicable. On the contrary, additional studies examining hepatocellular carcinomas, brain metastases and glioblastomas suggest that acetate may represent a key energy substrate for progression of such tumors. ${ }^{30,31}$ Indeed, in these models, suppression of acetate metabolism via inhibition of acetyl co-A synthetase 2 may represent a viable therapy. ${ }^{31}$ Therefore, while the nanoparticle delivery vector we have 
used represents a practical means of targeting tumorigenic tissue, selecting which therapeutic agents to encapsulate will depend on the type of malignancy being targeted.

\section{Conclusion}

We have manufactured and evaluated acetate-carrying nanoparticles that are preferentially delivered to tumors. Chronic administration of LITA-CAN led to reduced tumor growth in an HT-29 xenograft model of colorectal cancer, potentially via epigenetic modification. These data indicate that LITA nanoparticle systems, with appropriate modifications, are capable of altering the development of pathogenic signatures indicative of cancer.

\section{Disclosure}

The authors report no conflicts of interest in this work.

\section{References}

1. Donohoe DR, Collins LB, Wali A, Bigler R, Sun W, Bultman SJ. The Warburg effect dictates the mechanism of butyrate-mediated histone acetylation and cell proliferation. Mol Cell. 2012;48(4):612-626.

2. Zeng H, Lazarova DL, Bordonaro M. Mechanisms linking dietary fiber, gut microbiota and colon cancer prevention. World J Gastrointest Oncol. 2014;6(2):41-51.

3. Higgins JA. Resistant starch: metabolic effects and potential health benefits. J AOAC Int. 2004;87(3):761-768.

4. Kramer $\mathrm{OH}, \mathrm{Zhu} \mathrm{P}$, Ostendorff $\mathrm{HP}$, et al. The histone deacetylase inhibitor valproic acid selectively induces proteasomal degradation of HDAC2. EMBO J. 2003;22(13):3411-3420.

5. Soliman ML, Smith MD, Houdek HM, Rosenberger TA. Acetate supplementation modulates brain histone acetylation and decreases interleukin-1beta expression in a rat model of neuroinflammation. J Neuroinflammation. 2012;9:51.

6. Chiaradonna F, Cirulli C, Palorini R, Votta G, Alberghina L. New insights into the connection between histone deacetylases, cell metabolism, and cancer. Antioxid Redox Signal. 2015;23(1):30-50.

7. Waldecker M, Kautenburger T, Daumann H, Busch C, Schrenk D. Inhibition of histone-deacetylase activity by short-chain fatty acids and some polyphenol metabolites formed in the colon. J Nutr Biochem. 2008; 19(9):587-593.

8. Hamer HM, Jonkers D, Venema K, Vanhoutvin S, Troost FJ, Brummer RJ. Review article: the role of butyrate on colonic function. Aliment Pharmacol Ther. 2008;27(2):104-119.

9. Cummings JH. Short chain fatty acids in the human colon. Gut. 1981; 22(9):763-779.

10. Steliou K, Boosalis MS, Perrine SP, Sangerman J, Faller DV. Butyrate histone deacetylase inhibitors. Biores Open Access. 2012;1(4):192-198.

11. Allison SD. Liposomal drug delivery. J Infus Nurs. 2007;30(2):89-95. quiz 120.

12. Allen TM, Cullis PR. Liposomal drug delivery systems: from concept to clinical applications. Adv Drug Deliv Rev. 2013;65(1):36-48.
13. Hashizaki K, Taguchi H, Itoh C, et al. Effects of poly(ethylene glycol) (PEG) concentration on the permeability of PEG-grafted liposomes. Chem Pharm Bull. 2005;53(1):27-31.

14. Pirollo KF, Chang EH. Does a targeting ligand influence nanoparticle tumor localization or uptake? Trends Biotechnol. 2008;26(10): $552-558$.

15. Maruyama K. Intracellular targeting delivery of liposomal drugs to solid tumors based on EPR effects. Adv Drug Deliv Rev. 2011;63(3): 161-169.

16. Kenny GD, Kamaly N, Kalber TL, et al. Novel multifunctional nanoparticle mediates siRNA tumour delivery, visualisation and therapeutic tumour reduction in vivo. J Control Release. 2011;149(2):111-116.

17. Sahuri-Arisoylu M, Brody LP, Parkinson JR, et al. Reprogramming of hepatic fat accumulation and 'browning' of adipose tissue by the shortchain fatty acid acetate. Int J Obes (Lond). 2016;40(6):955-963.

18. Bhalerao SS, Raje Harshal A. Preparation, optimization, characterization, and stability studies of salicylic acid liposomes. Drug Dev Ind Pharm. 2003;29(4):451-467.

19. Dos Santos N, Allen C, Doppen AM, et al. Influence of poly(ethylene glycol) grafting density and polymer length on liposomes: relating plasma circulation lifetimes to protein binding. Biochim Biophys Acta. 2007; 1768(6):1367-1377.

20. Scheffler IE. Mitochondria make a come back. Adv Drug Deliv Rev. 2001;49(1-2):3-26.

21. Vander Heiden MG, Cantley LC, Thompson CB. Understanding the Warburg effect: the metabolic requirements of cell proliferation. Science. 2009;324(5930):1029-1033.

22. Seyfried TN, Shelton LM. Cancer as a metabolic disease. Nutr Metab (Lond). 2010;7:7.

23. Lunt SY, Vander Heiden MG. Aerobic glycolysis: meeting the metabolic requirements of cell proliferation. Annu Rev Cell Dev Biol. 2011;27:441-464

24. Munoz-Pinedo C, El Mjiyad N, Ricci JE. Cancer metabolism: current perspectives and future directions. Cell Death Dis. 2012;3:e248.

25. Roden M. How free fatty acids inhibit glucose utilization in human skeletal muscle. News Physiol Sci. 2004;19:92-96.

26. Qu Y, Zhang J, Wu S, Li B, Liu S, Cheng J. SIRT1 promotes proliferation and inhibits apoptosis of human malignant glioma cell lines. Neurosci Lett. 2012;525(2):168-172.

27. Bonnet S, Archer SL, Allalunis-Turner J, et al. A mitochondria-K+ channel axis is suppressed in cancer and its normalization promotes apoptosis and inhibits cancer growth. Cancer Cell. 2007;11(1): 37-51.

28. Babu E, Ramachandran S, CoothanKandaswamy V, et al. Role of SLC5A8, a plasma membrane transporter and a tumor suppressor, in the antitumor activity of dichloroacetate. Oncogene. 2011;30(38): 4026-4037.

29. Dang CV. Links between metabolism and cancer. Genes Dev. 2012; 26(9):877-890.

30. Yeung TM, Gandhi SC, Wilding JL, Muschel R, Bodmer WF. Cancer stem cells from colorectal cancer-derived cell lines. Proc Natl Acad Sci US A. 2010;107(8):3722-3727.

31. Schug ZT, Peck B, Jones DT, et al. Acetyl-CoA synthetase 2 promotes acetate utilization and maintains cancer cell growth under metabolic stress. Cancer Cell. 2015;27(1):57-71. 


\section{Supplementary materials}

LITA-CAN nanoparticle components
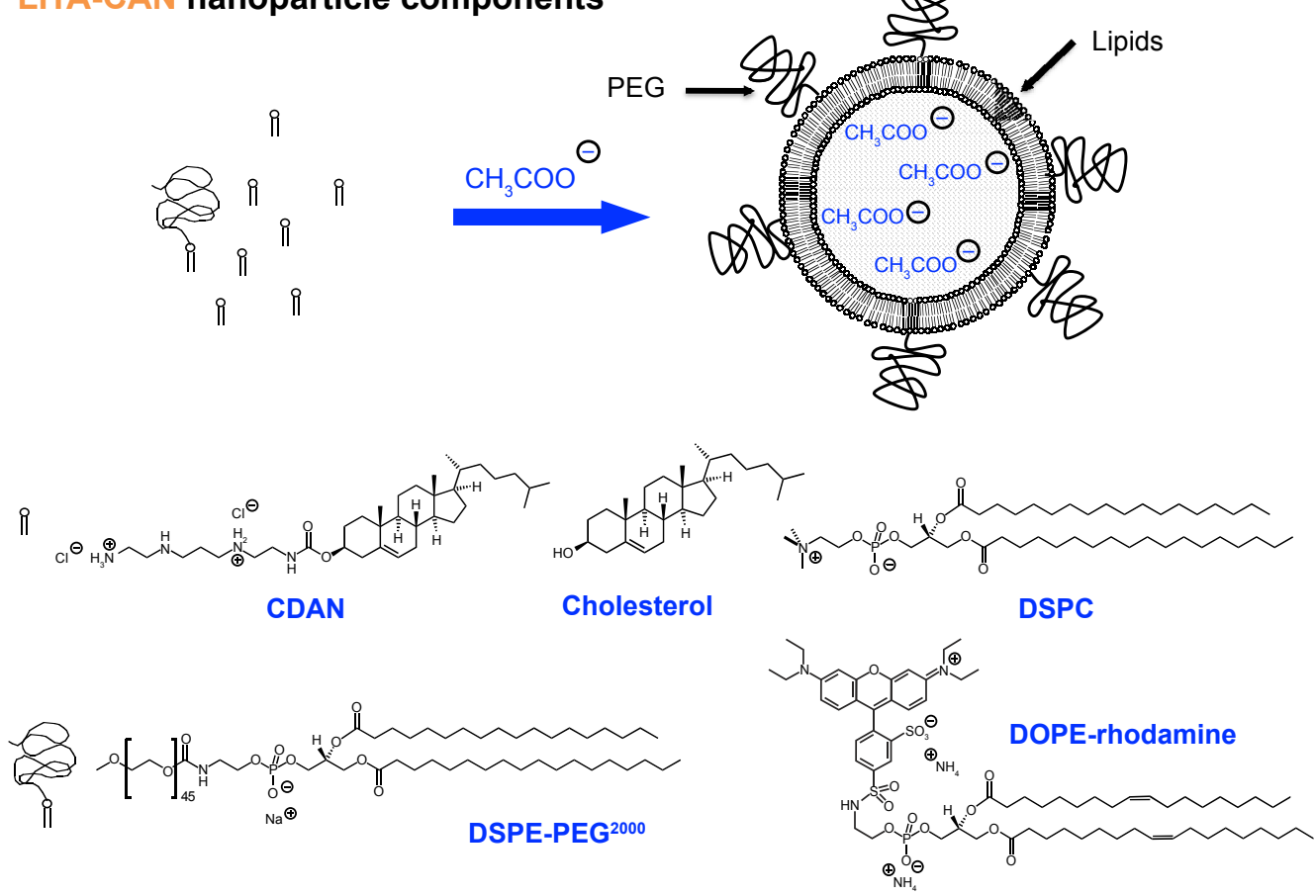

\begin{tabular}{|l|l|l|l|l|l|}
\hline Molar component (\%) & CDAN & DSPC & Chol & DSPE-PEG $^{2000}$ & $\begin{array}{l}\text { DOPE- } \\
\text { rhodamine }\end{array}$ \\
\hline LITA-CAN & 32 & 32 & 31 & 5 & - \\
\hline LITA-CAN-Rhd & 32 & 32 & 30 & 5 & 1 \\
\hline
\end{tabular}

Figure SI LITA nanoparticle design principles and percentage molar composition of formulations.

Abbreviations: LITA-CAN, liposome-encapsulated acetate nanoparticles for cancer applications; PEG, polyethylene glycol; CDAN, N'-cholesteryloxycarbonyl-3,7diazanonane-1,9-diamine; DSPC, I,2-distearoyl-sn-glycero-3-phosphocholine; DSPE, I,2-distearoyl-sn-glycero-3-phosphoethanolamine; DOPE-rhodamine, I,2-dioleoyl-snglycero-3-phosphoethanolamine- $\mathrm{N}$-(lissamine rhodamine B sulfonyl); LIP-CAN-Rhd, contains an additional I\% of DOPE-rhodamine for histological analysis.

Table SI Liposome-encapsulated acetate variant compositions and nanoparticle dimensions

\begin{tabular}{llll}
\hline Lipid & Lipid variation & Size $(\mathbf{n m})$ & Acetate encapsulated $(\mathbf{m M})$ \\
\hline LITA main formulation & CDAN/DSPC/Chol/DSPE-PEG ${ }^{2000}$ & $80-110$ & 4.41 \\
DSPC & DSPE & $35-80$ & 1.70 \\
& DLPC (CI2) & $35-150$ & 1.02 \\
& I0\% DSPC & $15-100$ & 0.51 \\
& $50 \%$ DSPC & $30-110$ & 0.00 \\
Chol & DC-Chol & $25-45$ & 2.79 \\
& I0\% Chol & $20-1,000$ & 0.00 \\
DSPE-PEG 2000 & $50 \%$ Chol & $20-160,1,000+$ & 0.00 \\
& DSPE-PEG500 & $0-150$ & 6.13 \\
CDAN & DSPE-PEG 10000 & $35-80$ & 0.03 \\
& DDAB & & 1.28 \\
\hline
\end{tabular}

Abbreviations: LITA, liposome-encapsulated acetate; CDAN, N'-cholesteryloxycarbonyl-3,7-diazanonane-1,9-diamine; DSPC, I,2-distearoyl-sn-glycero-3-phosphocholine; PEG, polyethylene glycol; DSPC, I,2-distearoyl-sn-glycero-3-phosphocholine; DSPE, I,2-distearoyl-sn-glycero-3-phosphoethanolamine; DDAB, dimethyldioctadecyl ammonium bromide; DLPC, I,2-dilauroyl-sn-glycero-3-phosphocholine; DC-Chol, 3ß-[N-(N',N'-dimethylaminoethane)-carbamoyl]-cholesterol. 


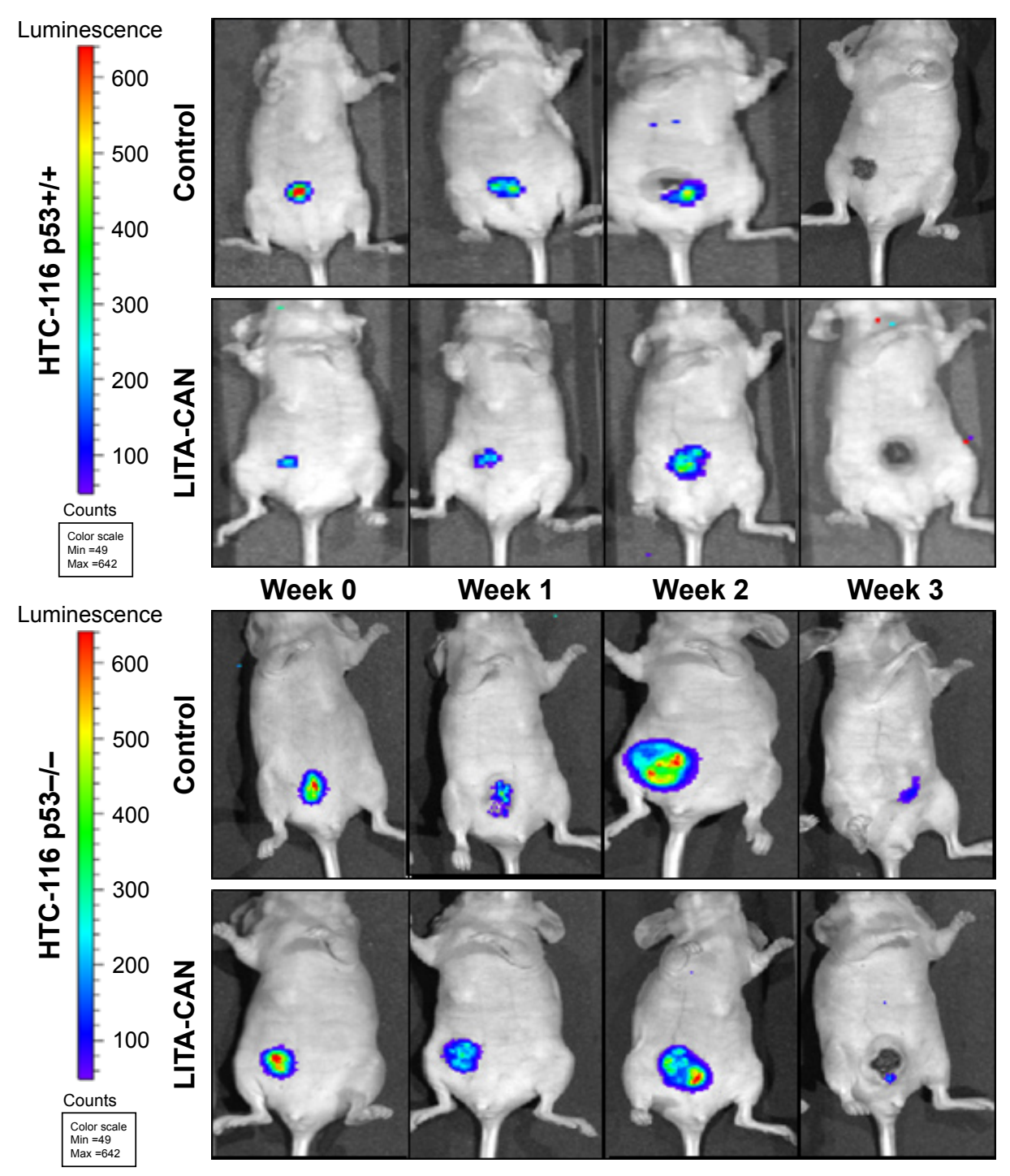

Figure S2 Bioluminescence imaging for murine xenograft models of HCT 116 p $53+/+$ and HCT 116 p53-/-.

Notes: Representative images of the murine xenograft model of HCT I I 6 p53+l+ and HCT I 6 p 53-I- after 3 weeks of chronic LITA-CAN or control (HEPES) nanoparticle i.p. injections. Color indicates luciferase activity, which is correlated with counts given on the scale.

Abbreviations: LITA-CAN, liposome-encapsulated acetate nanoparticles for cancer applications; HEPES, 4-(2-hydroxyethyl)-I-piperazineethanesulfonic acid; i.p., intraperitoneally.

\section{Publish your work in this journal}

The International Journal of Nanomedicine is an international, peerreviewed journal focusing on the application of nanotechnology in diagnostics, therapeutics, and drug delivery systems throughou the biomedical field. This journal is indexed on PubMed Central,

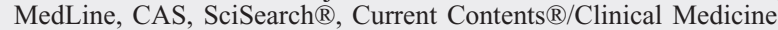

Journal Citation Reports/Science Edition, EMBase, Scopus and the Elsevier Bibliographic databases. The manuscript management system is completely online and includes a very quick and fair peer-review system, which is all easy to use. Visit http://www.dovepress.com/ testimonials.php to read real quotes from published authors. 